\title{
The Concept of Shi: Chinese Aesthetics and Happy Rain on a Spring Night (2004)
}

\author{
Nancy Yunhwa Rao
}

NOTE: The examples for the (text-only) PDF version of this item are available online at: https://www.mtosmt.org/issues/mto.20.26.3/mto.20.26.3.rao.php

KEYWORDS: Happy Rain on a Spring Night (2004), Chinese calligraphy, gesture, Chinese poetry, Du $\mathrm{Fu}$, Chinese aesthetics, motive, musical topic

ABSTRACT: The article brings a conceptual framework of Chinese aesthetics, music analysis, and theory developed in North America to bear on the analysis of Chen Yi's chamber ensemble Happy Rain on a Spring Night (2004). As a framework to analyze the work (both the poem by Du Fu and the music composition), it proposes the concept of shi, which can be described as "dynamic configuration" or "the probability and propensity that drive the process." The end of the article discusses issues related to culturally situated analysis.

DOI: $10.30535 / \mathrm{mto} 26.3 .0$

Received January 2019

Volume 26, Number 3, August 2020

Copyright $\odot 2020$ Society for Music Theory

\section{[1] Introduction}

[1.1] Concert music of the twenty-first century in America has become a fertile ground for cultural convergence. With unprecedented diversity, composers, performers, and listeners alike negotiate the rich sonic world based on their experiences, perspectives, encounters, and creativity. Scholars and theorists are no exception, as they endeavor to render meaningful interpretation and analysis of musical works. In this regard, intercultural compositions present a particular challenge. The traditional modes of musical analysis, which have been well-established in the field of music theory in North America and which generally place great emphasis on pitch content and form, do not seem to suffice. Byron Almén once wrote, "A single musical event can become a battleground for competing worldviews, methodologies, and social stances. Music, though a complex phenomenon, is often stripped of its complexity by the constraints of disciplinary boundaries and individual predispositions" $(2006,1)$. According to Almén, who advocates for multiple modes of analysis, we would do well to explore a range of valuable strategies for apprehending and organizing music phenomena to enrich our discourse. Scholars, indeed, have developed such a range of strategies, including semiotics, topic theory, narrative analysis, timbre analysis, and analysis of musical embodiment. These methodologies integrate analytical approaches that are discrete yet able to work together effectively to produce a balance of impulses. This article 
proposes such an approach in the analysis of contemporary Chinese music. My analytical method is informed by a deep-rooted connection to the aesthetics of Chinese classical tradition as well as my training in musical analysis and theory as developed in North America. This article will consider centrally a mode of speculation and explanation-shi-in tandem with the modes of analysis that model musical processes based on pitch structure.

[1.2] The notion of shi is an elusive yet important aesthetic concept in Chinese culture. The concept is particularly germane to works of contemporary Chinese composers. This article will begin with a discussion of the principles of this concept, and then explore its analytical implications for Chen Yi's Happy Rain on a Spring Night (2004), which is inspired by a classic poet of the Tang dynasty, Du Fu. In this famous poem of bucolic beauty, Chen Yi found a kindred spirit and brought into a contemporary frame the poetic spirit of the classical poem. Since the aesthetic of shi is of utmost importance in Chinese classic poetry and calligraphy, this work presents a fascinating opportunity to consider how shi in verses can be echoed in music. In my analysis, I propose a hermeneutic approach that engages two interpretive layers - realism and poetic shi-in both the poem and the musical composition. I argue that the seamless fusion of material realism and poetic elatedness is essential to Du Fu's poem, an aspect which resonates strongly in Chen Yi's work. Ultimately this analysis hopes to show the ways in which the work "unfolds progressively, from one stage to the next, becoming more fertile along the way," an important characteristic of shi (Jullien 1999, 124). In the concluding section, I will consider more generally the possibility of incorporating Chinese aesthetics in musical analysis.

\section{[2] Shi, Calligraphy, and Chinese Aesthetics}

[2.1] As a concept, shi (勢) is everywhere in Chinese culture, ranging from war strategy to Taoism to literature, painting, calligraphy, and music. The notion of shi is so omnipresent and familiar in Chinese society that words cannot fully explain it. When used in the context of visual art and music, shi often refers to the bringing together of form and momentum in a single expression, and is deemed an essential aesthetic concept. Yet it is also much more. A quick survey of its English translation provides a glimpse into multiple dimensions of this concept. In the Collins ChineseEnglish Dictionary, shi is translated as "force," "gesture" and "tendency." Elsewhere, shi is commonly translated as "potential," "power," "momentum," "vitalism," and "necessary imperative." Two translations are particularly astute and worth noting for their relevance to music. In a text about Chinese painting, shi is translated as "dynamic configuration" (Bush and Shih 2012, $21,165,353)$. In the context of the sociological thought of Laozi, shi is given this translation: "potentiality or propensity that seems to drive the process" (Robinson 2017, 130).

[2.2] Arguably the most explicit and visual representation of the elusive and abstract concept of shi is brushwork in Chinese calligraphy, a significant aesthetic that holds a special place in Chinese culture. The use of a brush as a writing instrument is essential to this ancient art form. As Chinese scholar Ronald Egan notes, "[A]lthough calligraphy does consist of written words, its meaning as art has never been restricted to the verbal meaning of the words that the calligrapher writes. The words function in calligraphy in a nonverbal way. . .Ideas and meaning [are] conveyed by calligraphic images" $(2004,282)$. From the Han to the Song dynasties, the art of calligraphy has developed several types of script (clerical, cursive-clerical, standard, running, cursive, wildcursive), engendering sophisticated and distinctive brushwork. Calligraphy masters made their consummate marks that distinguish their styles and lineage, as well as their unique contribution to the literati art. The notion of shi concerns particularly the movement of brush strokes - the key to an overall expression and aesthetic of the art-and by extension the calligraphy's "gestures [that] form a projection of the artist's body language," as noted by Wen Fong $(1999,29)$, the head of Chinese Painting and Calligraphy at New York's Metropolitan Museum. Chinese literature scholar Yu-kung Kao further explains, "As lyrical experience, calligraphy concentrates on the phase of execution, which is the materialization of the power of the artist. . .[P]erformance is [dependent] upon the inner reserve the artist can release at the moment. To appreciate calligraphy is to relive the physical action in one's mind" $(1991,80)$. These insightful commentaries underscore the 
prominence of inner energy and embodiment-important aspects of shi-in the aesthetics of calligraphy.

[2.3] In his description of the famous calligrapher Huang Tingjian (1045-1105), Wen Fong connects the essence of his work to shi: "[Huang's] large, bold running script is powerfully energetic: its gestures and forms may be read as the artist's body language-exuberant, even defiantly triumphant. . [H] e creates pure energy and movement through the momentum, or force (shi), of his brushwork" $(1999,48)$. In other words, the maneuvering of the brush as it discharges its characters in a fluid line derives from a kind of propensity, a release of inner energy that drives the momentum of the line and creates the particular aesthetic qualities of the character. Moreover, brilliant calligraphic work brings together the stroke, line, shape and meaning, all in one creative impulse, as demonstrated in Example 1. Not only does each character have its shi at work, but the shi also continues vertically from one character to the next as if a breath runs through the full column.

[2.4] Video Example 1 shows a calligrapher at work, writing the first three verses of a Songdynasty poem by Su Shi, "Nian Nu Jiao: Memories of the Past at Red Cliff." The three verses read, "The great river flows east / Its waves scouring away / The dashing heroes of a thousand ages" (Egan 1994, 226). We can observe how in single continuous physical movements the calligrapher composes the strokes of each character with deft pivoting actions of his fingers and wrist. The flicking and turning brushstrokes of varying thickness, shape, speed, and pressure form each character in a calculated balance. The artist attends to the continuity of flow as well, with the brush moving fluidly from one character to the next along the vertical column. The notion of shi is most explicit at the 1:13 mark where he retraces the last stroke in the just-written character, picking up the gesture and flow in order to continue the motion in the new character.

[2.5] An expression of shi is also seen in the continuation of the brush strokes when the brush is no longer pointed and supple with ink. As the semi-dry brush renders strokes in sparse ink, making visible ample white paper underneath the strokes, it imparts no less a flow of energy than when it is fully inked, giving the character drive and cohesiveness. The force is continued through. In summary, an important aspect of shi is the force of vitality that runs through the brushwork, where it is made to be felt through the balanced strokes and the space around them. It lives within the characters in self-contained ways. Or as Kao notes, it is "a course through which the torrent flows" $(1991,80)$.

[2.6] Another important aspect of shi is the notion of "disposition." French philosopher François Jullien (1999) posits that shi involves three elements: the disposition, the set-up and the line. They work together to carry out the dynamism of shi. He writes, "On the one hand there is the disposition of things - their condition, configuration, and structure. On the other there is force and movement" (11). This postulation foregrounds the key role of disposition: shi is the potential dynamic and inner energy born of a particular disposition, a beginning. In other words, the dynamic and strategic disposition generates "an ineluctable unfolding given shape by propensity" (247). Importantly, then, shi not only expresses an inner energy from which the outer form is materialized, but also comprises a configured positioning from which it exerts the force that unfolds and releases inner energy. All starts from an inclination.

[2.7] The concept of shi, an aesthetic force and a fundamental intuition in Chinese culture, can be felt in the music of many contemporary composers. In an essay on Chen Yi's Second Symphony, I analyze the composer's use of Peking opera's percussion music, the rhythmic patterns in particular, to create dynamic force (Rao 2016). The analysis connects this dynamic musical force to Robert Hatten's notion of gesture, "energetic shaping of sound through time" $(2004,93)$, as well as Arnie Cox's $(2001,2011)$ idea of mimetic hypothesis - that is, an experience of music involving an imitation in the listener's mind of bodily exertions. The approach brings into focus a prominent aspect of this symphony, namely, the inner energy (created by allusions to traditional percussion patterns in Peking opera) that shapes the musical gestures, as well as the embodied listening these musical gestures elicit. In that essay, I briefly touch on the notion of shi to underscore the prominence of this energetic shaping and embodiment. Ultimately, the analysis makes explicit the inner energy running through the musical gestures, an important part of the symphony's appeal. 
[2.8] Without using the term, shi, Yayoi Uno Everett (2007) nevertheless pays close attention to some of its inherent elements - such as energy, oneness, gestural continuity -in her analysis of Chou Wen-Chung's application of the concept of calligraphy in his compositions String Quartet No. 2 and Windswept Peaks. ${ }^{(1)}$ She also demonstrates how the calligraphic principles generate the formal and musical designs in Chou's work. For example, she notes how the use of glissando, variable modes (created by Chou), and instrumental timbre to shape initial thematic entries emulates the compound circular movement at the start of a single brushstroke, and that the "spiral motion [from the passing of motives] occurs as variants of invisible motion between strokes" (582). Since the composer explicitly creates musical gestures modeled after various techniques and attributes of calligraphic brushstrokes, the concept of shi is necessarily present even if unstated. ${ }^{(2)}$

[2.9] In this essay, I will focus on the role of shi as an aesthetic concept in Du Fu's Happy Rain on a Spring Night and Chen Yi's eponymous work. This analysis will consider the manifestation of shi in several dimensions. In one of the earliest texts on Chinese painting and calligraphy, the treatise Notes on Brushwork, the noted scholar and calligrapher Ching Hao (c. 855-915) writes, "The different appearances of mountains and streams are produced by the combinations of vital energy (氣 chi) and dynamic configuration (勢 shi)" (Bush and Shih 2012, 164). He names four different types of force (shi):

There are four types of forces (shih) in brushwork - muscle, flesh, bone and spirit. If a stroke with thickening and thinning width is filled with substantial inner force, its force is called "flesh." If a stroke is vigorous and upright, with force to give life to dead matter, its force is called "bone." If each stroke delineating the whole painting is undefeatable, the total force is called "spirit." (Bush and Shih 2012, 171)

The four categories help delineate the varied forms of shi, of which the notion of "spirit" will be adopted for this analysis. In the following discussion, I will examine shi and "spirit" in Du Fu's poem - the poetic shi-and consider its manifestations in Chen Yi's composition - the musical shi.

\section{[3] The Poem: Layers of Realism and Inner Dynamism}

[3.1] Du Fu (712-770), a prominent poet and civil servant during the Tang Dynasty, wrote the bucolic poem when he was 51. After a long period of turmoil due to wars and political persecution, he settled in Chengdu and established a residence named Huanhua Cottage, a place that has been memorialized ever since. There he took to the rustic lifestyle and became very close to the peasants. It was one of his most productive periods. Lauded for his innovative expansion of topical choice and poetic effect, Du Fu was also known for his compassion, his plain and direct depiction of daily life, objects and surroundings, and his prosodic style (S. Chou 1991). His penchant for nature, materiality, and realism kept him a celebrated poet long after the Tang Dynasty. His signature genre, known as "poetry on objects" or yong-wu poems, focuses on materiality and on subjects such as animals, plants, mountains, and rivers. The subject matter is unpretentious and the depiction plain. As Du Fu scholar Shan Chou noted, "The specific trait original with [Du Fu] is a kind of realism based on an acute sense of everyday life, very physical and concrete in its expression. Physicality lies at the heart of this realism" $(1991,21)$. Natural images such as bird, rain, and flower are the staples of description in Du Fu's poems. Below is the Chinese version, romanization, and an English translation of the poem Happy Rain (the title is translated here as Spring Night, Delight in Rain). To hear the entire poem read by Chen Yi, please click on Audio Example 1.

$$
\text { 春夜喜雨 }- \text { 杜甫 }
$$

(romanization)

好雨知時節，當春乃發生。 hao yu zhi shi jie / dang chun nai fa shen/

隨風潛入夜，潤物細無聲。 sui feng qian ru ye / run wu xi wu sheng/

野徑雲俱黑，江船火獨明。ye jing yun ju hei / jiang chuan huo du ming/

曉看紅滋處，花重錦官城。xiao kan hong shi chu / hua zhong jin guan cheng/ 
The good rain knows when to fall,

stirring new growth the moment spring arrives.

Wind-borne, it steals softly into the night,

nourishing, enriching, delicate, and soundless.

Country paths black as the clouds above them,

on a river boat a lone torch flares.

Come dawn we'll see a landscape moist and pink,

blossoms heavy over the City of Brocade.

[3.2] Happy Rain is a celebrated yong-wu poem that beautifully illustrates the principles of the genre. From the first line, this poem presents descriptive details of many ordinary matters. We learn of the landscape, with its wind, night, path, cloud, river, boat, light, flowers, and city. The depiction of objects is also infused with an attitude of fondness and playfulness toward them. Du Fu avails himself, as he often does in such poems, of personification, which allows him to turn elements of nature into joyful playmates. Here, the rain is made a character, one that is pleasant and beneficial to the world, timely providing moisture for creatures and crops in spring. In each of the first two couplets, a phenomenon is presented first, and then its cause is rendered: the rain is pleasant and welcome, because the spring is a time of growth; the night rain seems to steal in with the wind, because it quietly and gently moistens the crop. The inversion of cause and effect reinforces the agency of the rain, making it an active character in the scene, and lends a dynamic quality to the imagery of spring rain.

[3.3] In the third couplet, the poem moves from the active and sonic to the descriptive and visual. The dark land and path wet from the rain make the lone torch flame of the river boat glow. In the fourth and last couplet, the poem visualizes the colorful, brilliant scene of flowers all over town at daybreak. The imagery of these two couplets suggests a temporal movement from the night to the dawn, and a spatial change from the natural field to the city of refinement and sophistication. The underlying shifts in time and space enrich the images of the poem, adding complexity to what appears at first to be a simple depiction of rain. At a syntax level, the action mobilized by the verbs in the first two couplets stops at the third couplet, which contains no verbs (in the Chinese version), only to be set free by the busy scene implied in the fourth couplet. ${ }^{(3)}$ These individual actions and elements in the verses constitute a first layer of the yong-wu poem, the "layer of realism," of the surrounding world. It is the configuration of reality.

[3.4] Meanwhile, as the poem proceeds with meticulous descriptions of the spring rain, animating its feel, wit, sound, and movement, it also poignantly conveys the mood of joy and a sense of graciousness and pellucidity. The poem is infused with a deep-seated yearning for the spring rain, and thus a sense of elatedness with its arrival. The poem's focus on ordinariness of subject matter brings out the extent of Du Fu's empathy with common folk, portraying their sheer exuberance at the arrival of rain just in time for the season of growth. The plain depiction vividly conveys the spirit of compassion.

[3.5] What propels the poem's sense of elatedness is a layer of force and inner dynamism. It can be described as the poetic shi, the emotion of the poem. This emotion is not stated directly in the verses, nor does the actual word "happiness" appear anywhere. Yet the spirit is clearly felt. The poet's rhythmic crafting of the verses aides the emergence of the poetic shi, a dimension of vital inner energy that is palpable throughout the poem. (A reading of the poem in individual couplets by the composer can be found in Chen's article in this issue, Audio Examples 1-3.)

[3.6] Generally, in this standard eight-line or four-couplet poetry genre of the Tang Dynasty, the first couplet provides the theme and the disposition. The second couplet sets the opening theme in motion and begins a full development of the dynamism. The third couplet provides "turns," a kind of change. And in the last couplet, "there is a return to the more [... ] expressive mode to bring the poetic act to an end that reverberates with the poet's actual surroundings" (Kao 1991, 74). Thus the second couplet is pivotal in terms of movement. The above translation of the first two couplets captures the melodic and rhythmic flow of the Chinese version particularly well. The second 
couplet presents actions that set the opening theme of the first couplet in motion, creating a sense of development. It dramatizes the good rain, achieves a fuller sense of bliss, and carries the poem forward. Through the flow, the unfolding of poetic shi animates and articulates the emotion and state of mind. This layer of dynamic force is connected to what is commonly seen in Chinese poetry - "human consciousness," the correspondence between mind and the state of its surroundings. ${ }^{(4)}$

[3.7] Together, the layer of realism and the layer of force and inner dynamism (the poetic shi) contribute to a fusion of the scene and the feeling. There is, as Jullien notes, the "configuration of the dynamism inherent in reality" $(1999,75)$. Du Fu's poem succeeds beautifully in conveying the poetic shi, the vivid spirit, moving from the general context to the matter itself. With the strong sense of progression delineated in the material and the landscape, Du Fu creates a force of optimism, to which Chen Yi responds musically in profound ways.

\section{[4] The Music: Layers of Realism and Poetic Shi}

[4.1] In this chamber work for five instruments (flute, clarinet, violin, cello and piano), Chen Yi

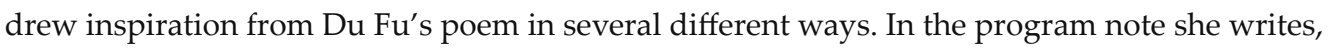
"The music reflects the scenes and the expression according to the meaning of the poem when it's being unfolded line by line" (2014). Indeed, the poem serves as the impetus for numerous musical designs, from formal structure and pitch content to gestures, timbre, dramatic tension, texture, and so forth. To begin, Chen Yi apportions the four couplets of the poem within the bipartite form outlined in Example 2. In Part I, sections A and B draw from the first two couplets of the poem; sections $C$ and $D$ reflect the change to a darker landscape in the third couplet. The first part also includes section E, the beginning of the toccata sections that reflect the fourth couplet. Part II includes the toccata sections F and G that draw from the grand mood of the fourth couplet, and section $\mathrm{H}$ is the coda that brings back elements from previous sections. A notable switch to a new timbre in section $\mathrm{E}$, corresponding to the beginning of the fourth couplet, reflects the dramatic change from the dark night in the fields to the colorful flowers at daybreak in the city. Unlike the earlier texture, section $\mathrm{E}$ is distinguished by the piano's dense and constant percussive toccata-like rhythm. It generates a mood reflecting the shift to, in the composer's own words, "the new future." As in many of Chen Yi's other works, the principle of the Golden Section plays an important role, determining the duration of each section in this two-part structure. The Golden Mean falls on section $\mathrm{F}$, the beginning of the climax of the piece, which is high in dynamic level and energetic as a whole. All subdivisions follow proportions determined by the principle of the Golden Section, and the texture changes accordingly throughout.

[4.2] Chen Yi's rendition of this Tang-dynasty poem is a convergence of classic poetry and musical ideas. In what follows, I will consider the two layers of the poem - realism and inner dynamismas they unfold through a range of sonic imagination. From this perspective, I argue that, on the one hand, Chen responds to the poem's naturalistic depiction of realism by foregrounding certain motives, gestures and rhythmic figures to produce a vivid portrayal of the natural landscape. On the other hand, she creates the sense of elatedness present in the poem by generating a strong sense of momentum in the sonic space through distinctive musical designs. The aesthetic effect of this composition as a whole, therefore, results less from tunes that lead the listeners and more from gestural movements that project the emotional content-as in the shi of brushstrokes in calligraphy. The interaction between layers of realism and poetics is most clearly articulated in sections A and $\mathrm{B}$, which will be the focus of the analysis in the next two sections.

\section{(1) Three Musical Images of Realism}

[4.3] Corresponding to the first two couplets, Sections A and B comprise three musical imagesprimarily distinguished by their timbre and gestures - that I would characterize as conveying the sense of materiality depicted in Du Fu's verses. Readers will note that my analysis here differs significantly from the analysis that Chen Yi offered in her own discussion of this piece in this journal. ${ }^{(5)}$ These musical images are derived from my analysis of the work, although they can be connected to terms in the composer's picturesque description of the work. Each image is a musical 
depiction of a natural element implied in the poem's scenery. The first one is noted in Chen Yi's own introduction to the score, the budding seeds:

It's like the welcome rain on a quiet spring night that nurtures the budding seeds, our new society is pushing us forward to the new future. (2014)

This vivid musical image sonically connotes the growth of budding seeds: a brief melodic figure in the cello, shown in Example 3, suggests the spirit of quirky sprouting and proliferation. It consists of two fragments, both in fast rhythmic figures to provide a sense of sprouting. The two fragments are separated by rests, resulting in a temporal division of the melodic figure into three. A sense of symmetry is felt from the roughly mirrored contour of the chromatic steps of the two fragments. With each appearance, the melodic figure constitutes a quick and brief ascent, as if the flick of a brushstroke, a sprouting imagery. The budding-seed motive is twice transposed up by a minor third; the same sequence will also repeat in the violin. With each statement of this ubiquitous figure, a strong sense of materiality is projected by the metallic timbre of the tremolo and sul ponticello. The transpositions up by a minor third give a subtle ascending direction to the first part of the piece, as shown in Example 4. Two features of this musical figure make it stand out: the jagged and rough timbre and its rocky nature. Upon first encountering it, the listener is likely to note its shape, and its sequential reappearances create a landscape of scattered rugged sonic elements.

[4.4] The second musical image, which I call misty rain, is made up of blocks of dyadic tremolo in the woodwinds, as seen in Example 5. Its soft timbre conjures up the image of mist and drizzle. The misty-rain tremolo figure builds on a whole-tone tetrachord $\{C \#, E b, F, G\}$, made up of a tritone dyad in the flute and a whole-tone dyad in the clarinet, or vice versa. The same misty-rain image appears eight times, after which it moves up by seconds, reinforcing the build-up to the first climax in the work. This musical image relies on the gesture of tremolo, blending the timbres of flute and clarinet, as well as the pitch content. Both contribute to the mistiness of the image-a soft hue. It is gentle and quiet, leading the listener to hear it as part of the atmosphere of the landscape. The third musical image consists of short piano figures in the high register, shown in Example 6. In sixteenth-note quintuplets, this figure begins characteristically with two-octave leaps a whole tone apart, followed by brief flurries of chromatic steps that sometimes imitate the first musical image. The resemblance in pitch content between the third image and the first is certainly present, yet their timbre profiles could not be more different, forming two distinct gestures. For each of the musical images, timbre is an important part of its identity. In the piano's third musical image, its quiet percussive timbre suggests another element in the scene, the quiet raindrops. ${ }^{(6)}$

[4.5] These three musical images appear repeatedly in rather stable timbre and pitch structures. As shown in Example 7, they are interspersed with one another to shape the surroundings and the landscape. Temporally, a subtle sense of expansion is created through the gradual increase of the durational span between successive beginnings of the misty-rain image. Growing by a quarter note with each appearance, the span augments steadily from 9 to 12 quarter notes in Example 8. The musical process thus unfolds through time, much like a long horizontal scroll of Chinese landscape painting that can be viewed section by section, as if traveling through the landscape. ${ }^{(7)}$ The layer of realism is reflected in the musical configuration of the landscape, through which the dynamic and inner energy will emerge. Although they are most prominent in sections A and B, the three musical images reappear throughout the work, sometimes deeply buried in dense and thick textures, other times peering through the busy texture with their unique timbres. The images associated with them-budding seeds, misty rain, and quiet raindrops—-form a constant in a work that clearly has another dynamism driving its inner motion.

\section{(2) The Poetic shi as Musical shi}

[4.6] A dynamism can be felt from the start of the composition. The animated opening bars constitute an energetic gesture: the sprinting of quick ascending scales in woodwinds and piano, ending with the flute's trill on a high G6. This swift upward gesture (shown in Example 9) is the disposition of the dynamic force. (This disposition gesture is identified by Chen Yi in her discussion as A1.) The positioning of the opening gesture sets up and creates the capacity, the 
vitality of the shi, whose potential force will be activated in the course of the composition. In other words, these scalar ascents at the opening of the piece function as the disposition from which the momentum and dynamic force of elatedness-the poetic shi-will be born.

[4.7] Following the disposition, a significant dynamic force begins: a series of four large-scale momentum-building gestures based on ascending motion. The first one of these, Ascending Motion I, is a long gradual ascent in the muted violin, starting in m. 3, as shown in Example 10-I. (This gesture is described by Chen Yi in her article as A2.) The violin, playing tremolo, rises steadily in chromatic triplets from G3 to $\mathrm{C} \# 7$, spanning three and a half octaves in seventeen measures. A sense of inner energy and momentum is built up progressively, propelled by the violin's relentless upward motion. Although the initial six-note figure is transposed up by a semitone, the continuation uses a variety of chromatic melodic figures, avoiding anticipation of a regular recurrence of the melodic unit. The long ascent, though soft in dynamics, produces the first large-scale momentum.

[4.8] The violin's gesture is enhanced simultaneously by a separate ascent in the cello. When the opening flute reaches G6 in m. 2, the cello picks up the same pitch. In the sparse texture the cello's sustained G6 in harmonics is resounding. The cello first sustains G6 for three and a half bars. Only in $\mathrm{m}$. 6 does the cello begins to move up, joining Ascending Motion I that has already begun in $\mathrm{m}$. 3. There in $\mathrm{m} .6$ the ringing harmonics in the cello rises in a slow glissando from G6 to C\$7, which elegantly provides a framing for the ascending motion, anticipating the goal that the steadilymoving muted violin (of Ascending Motion I) will reach ten bars later. Listen to these processes in the audio that accompanies Example 10. The cello's musical process constitutes Ascending Motion II, as shown in Example 10-II.

[4.9] These two ascending motions, both rising to $\mathrm{C} \# 7$, confirm the potential of the opening disposition. They are followed by similar motions in different instrumental groups. After the muted violin reaches $C \sharp 7$ in $\mathrm{m} .18$ and sustains, the woodwinds take over in $\mathrm{m}$. 20 by initiating a new ascending motion with quintuplet figures initially centered around $C \sharp 4$. This constitutes Ascending Motion III, as shown in Example 10-III. The quintuplet melodic figure is played alternately by clarinet and flute, again avoiding obvious motivic patterns. The woodwinds rise continuously through two octaves over six bars, reaching the high Eb6. Overlapping with the woodwind's final $\mathrm{Eb} 6$, a new ascent begins in the muted cello in Example 10-IV. This is Ascending Motion IV, the cello's restatement of the violin's Ascending Motion I starting an octave lower on G2. The cello steadily rises for eighteen bars, reaching its peak at the end of section B, where many shorter ascending motions join to reinforce the upward drive to the piece's first climax. Ascending Motion V (shown in orange in Example 11) is a composite of these smaller ascending figures, creating the effect of a powerful soaring flight: the piano adds the sprout of the ascending scale sweeping to the top register; several blocks of woodwinds' misty-rain tremolo reappear in sequence (by ascending seconds), the third statement of which reaches $\mathrm{C} \# 6$, matching the goal of the steady long-range ascending motion of the muted cello. Finally, at the close of the climax, the muted violin in harmonics takes over again to sustain the high $C \sharp 7$ into the next section.

[4.10] Example 11, a reduction of sections A and B, summarizes how the opening bars establish the musical shi. Using colors to distinguish the gestures of the different instruments, the example shows how the passage consists of a series of overlapping ascending motions. Because each ascent begins before the previous one has finished, a continuous sense of acceleration and excited anticipation develops. These multiple forces of ascent engender a vital and soaring energy that rushes forth from the very first upsweeping gesture. This energy is made especially evident by the strategically placed repeated high $C \mathbb{B}_{\mathrm{s}}$, which are animated as the termini of gestures with different timings and timbres. ${ }^{(8)}$ Thus, the momentum of ascent portrays an inner energy that manifests itself in a variety of configurations.

[4.11] Collectively, these overlapping ascending motions portray a dynamism that articulates a growing feeling of elatedness and high spirits. A phrase from Jullien's discussion of shi best describes this linear and temporal dimension of the poetic layer of the music: "At precisely the point where the next shi arises, the preceding shi breaks off" $(1999,129)$. Throughout Sections A and $\mathrm{B}$, the sense of continuous dynamic momentum invigorates the work with a sense of the inner 
energy. Ascending motion is commonly understood as a form of building momentum and energy, and is related to elatedness, which signifies a mood of being uplifted, a swelling of emotion, feeling upbeat and high spirits. In the music, this is portrayed by musical motion that moves repeatedly from low to high. It gives a sense of becoming elated. Thus the poetic aesthetic-elatednessexpressed in $\mathrm{Du}$ Fu's poem is elegantly articulated here through the musical shi. The inner dynamism can be felt through the endless upward motion.

[4.12] Although the realism and poetic layers are discussed separately, their effects are far from isolated. Indeed, the interplay between the three musical images (representing the realism layer) and the general ascent (the poetic layer) constitute the work's main expression. As part of building up to the climax at the end of section B, the two domains are joined together to form a large ascending motion. In mm. 36-42, the misty-rain musical image moves up by a major second twice, such that the arrival of the cello's ascent at $\mathrm{C} \# 6$ is doubled by the flute's tremolo playing the same pitch. The musical image and ascending motion converge; they become one. Through Chen Yi's carefully chosen pitch structures, musical figures, and designs of ascent, the spirit of wit and optimism that distinguishes Du Fu's poem is made palpable.

\section{[5] Chinese Aesthetics and Music Analysis}

[5.1] Chinese classic poetry, calligraphy, painting, and aesthetics have had an enormous effect on the works of Chinese contemporary composers. The quintessence of this aesthetics is its sense of motion with dynamic life, which in calligraphy, for example, is registered as traces on paper, with time and rhythm in shifting space. Chinese composers maintain an underlying identification with, and draw inspiration from, this cultural heritage, creating works that resonate, either explicitly or implicitly, with principles inherent in the artistic tradition. Chen Yi's Happy Rain is an excellent example. What lies at the heart of such an aesthetic encounter (the ways composers transform, subvert or change the artistic tradition through new modes of expression) is the composers' individual practice, a kind of artistic mark similar to those "painterly marks" in the brushstrokes of Chinese calligraphy, as discussed in [2.2]. In considering such compositions, many questions arise. What are the different ways in which the effect of Chinese aesthetics may be felt in a musical work? How might listeners relate to the aesthetic aims of such musical compositions? And how essential is knowledge of Chinese aesthetics to the appreciation of the musical work? These questions inevitably arise in the minds of scholars, audiences, critics and performers, who themselves bring to the work a wide range of lived experiences and cultural inheritances. While these questions are not unique to the study of Chen Yi's music, the analytical approach used in this essay foregrounds these questions. With Happy Rain, Chen Yi found a kindred spirit in this famous yong-wu poem by $\mathrm{Du} \mathrm{Fu}$, and brought into a contemporary frame the poetic spirit of the classical poem through various musical idioms. Using the concept of shi as a lens, this analysis seeks to explore the poetic aesthetic in her work. It thus brings into focus the issues of disposition and inner energy, and the dynamism that carries that energy forward continuously in this composition. In a recent essay, Chen Yi refers to the significance of such disposition, noting the inspiration from calligraphy: "In calligraphy, every stroke begins with a point. After you set down the first dot, you turn the brush to make the different strokes that you want; so, the point, the beginning of each stroke, is very important in drawing Chinese calligraphy. . .My pipa solo piece The Points uses an idea like this" (2019). Similarly in Happy Rain, the gesture of ascending motion at the opening has the role of disposition, namely the initiation of shi, from which "the work unfolds progressively, from one stage to the next, becoming more fertile along the way" (Jullien 1999, 124).

[5.2] Other aspects of Chinese aesthetics can be found elsewhere in the composition. Examples abound, but two in particular stand out, both involving narration and recitation in a pronounced Chinese style. In the first example, a Western instrument emulates the distinctive, narrative sound of a traditional Chinese instrument. At the start of section C, the violin in harmonics and the cello in its lowest register jointly play a soulful tune in the form of a compound melody, shown in

Example 12. The high, thin timbre of the violin strikingly resembles the nasal sound of the Chinese erhu, a two-string bowed instrument known for its own close relation to the sound of the human voice. The solo erhu is often associated with very expressive and melancholy music, and listeners 
typically understand an erhu melody to be telling a story. The narrative characteristics of the melody in Happy Rain are projected not only by its soulful, expressive nature, full of subtle melodic gestures and phrasing, but also by its pitch content. The tune is saturated with the pentatonic tetrachord 0247, creating a strong aural link to the pentatonic folk music found in Chinese and many other cultures. As the violin and cello narrate the story in the deeply-felt emotional tones of the erhu, sharp punctuations from the piano and breathy key slaps from the flute enhance the mood. The melody effectively captures the atmosphere of the poem's third couplet, portraying the moonless night in which the lone torch on a boat seems the only source of illumination.

[5.3] Another example of Chinese aesthetics is the use of string instruments to emulate the tonal inflection of Mandarin Chinese in the recitation of the third and fourth couplets. With this technique, the composer pays homage to the familiar tradition of poetry recitation and chanting, a performative tradition associated with classic poetry. Classical Chinese poetry was traditionally studied and appreciated not only by silent reading but also through the practice of recitation and chanting. Grasping the rhymes, rhythm and sound patterns of words through recitation is part of learning the poem. In rhythmically more elaborated ways - namely, each syllable assigned widely varying durations - poetry chanting is valued as an art that enlivens the aesthetic nuance of a poem and helps the chanter and listeners to savor the auditory effect and emotional subtlety of classic poetry. ${ }^{(9)}$

[5.4] The technique first appears at the end of section C in mm. 65-69, shown in Example 13, where the cello recites the third couplet. The quick sliding tones suggest the changing pitch of tonal inflection, while the shifting dynamics, large leaps, and wide registral span further contribute to the effect of dramatic recitation. That the third couplet is being recited here is suggested by the register and timbre. The cello's low register and distinctive timbre even in higher registers - thick, heavy, dark - reflect the imagery of the third couplet: the night, the dark land wet from rain, and the lonely torch light. As the couplet contains two verses, the cello's recitation contains two phrases, each beginning low with a grace note and gradually ascending to the high point. The technique appears again at the start of section $\mathrm{G}$ in $\mathrm{mm}$. 134-141, as seen in Example 14, in which the violin recites the fourth couplet. The earlier sliding tones are now specified as glissandi, which articulate individual words to emulate the tonal bending of what is known as "heightened speech" in Chinese opera tradition. ${ }^{(10)}$ The effect of recitation is again enhanced by rapidly changing dynamics, large leaps, and a wide span. As in the cello passage, the violin's recitation is divided into two phrases, each beginning with a low grace note and gradually ascending, again corresponding to the two verses of the couplet. Now, however, the violin's higher register and its own distinctive timbre - thin, clear, bright - reflect the imagery of the fourth couplet: the arrival of dawn, the colorful and delicate blooms, and the poem's general sense of elatedness. These reciting "voices" produce the linguistic attributes that are an important part of the composition's poetic gesture.

[5.5] These two examples show different ways that Chen Yi draws inspiration from Chinese aesthetics and source materials. The difference between them is instructive. In the latter example the composer draws inspiration directly from a source-recitation of verses. ${ }^{(11)}$ Through strategically placed sliding tones and large leaps, the strings emulate the tonal inflection and other linguistic features of recitation. This is a direct use of source material-amounting to a kind of musical quotation - and the composer makes clear her intention and the desired effect by way of program notes or explanation. Such an approach is characteristic of Chen Yi's work, as seen in her frequent vivid use of melodies from Chinese folk traditions. On the other hand, the example of the soulful tune that emulates narration on an erhu does not draw directly from one source, nor has the composer mentioned a source for its inspiration. Yet the sonority is unique and striking, creating a perceptually "marked" event, a notable moment, in the piece. Its timbral effects, tone color and pitch characteristics conjure up a Chinese instrumental sound. And for listeners familiar with the tradition, its resemblance to the $e r h u$ is unmistakable. ${ }^{(12)}$ This is an allusive use of source material, but it is not difficult to infer the cultural reference from the sonority. As such, it conveys the Chinese aesthetic in a way that transcends the bounds of the source material and exists in the conceptual or subconscious realm. ${ }^{(13)}$ 
[5.6] Examples of both direct and indirect effects of Chinese aesthetics can be heard frequently in Chen Yi's oeuvre. At the same time, it is important to note that appreciating Chen's music by no means relies on knowledge of its source materials or Chinese cultural references. Her works may also be appreciated from the perspective of global contemporary art music. This is made amply evident by the critical acclaim she has received on international concert stages in the past two decades, from critics and colleagues who mostly do not share her Chinese heritage. ${ }^{(14)}$ Many of her compositional traits transcend cultural boundaries: the dynamism, the palette of sophisticated timbres and colors, well-balanced formal structures, tight-knit motivic connections, coherent harmonic language, and engaging musical narratives, to name a few. In other words, Chen Yi's work needs no "translation." Yet, by examining them through the lens of Chinese aesthetics and practices, layers of meaning and cultural significance that might otherwise go unnoticed are revealed. Indeed, it would be a missed opportunity not to engage with the cultural frame and reference network with which Chen Yi's musical imagination also resonates. To the extent that analyses of music inevitably rely on previously spoken words and phrases to communicate, bringing in the language of Chinese aesthetics and its concepts to discuss ideas pertinent to the composition seems not only befitting but also necessary. Chinese perspectives matter as much to historians, audiences, and performers as to analysts and theorists. Thus, situating analyses of such compositions within Chinese aesthetics is important because it engages with the multivalent cultural context of a work's genesis, enabling a deeper and more complete understanding of the music. Such analysis provides an opportunity to comprehend Chen Yi's artistic imprint and her individual practice, and, just as significantly, to expand our modes and languages of musical analysis to be more in line with the cultural complexity of musical compositions today.

[5.7] The same could be said of the more abstract notion of Chinese aesthetics, shi. Deeply entrenched in Chinese culture, the concept of shi exists both as a guiding principle for artistic expression and subconsciously as a way of being-that is, the human consciousness. To analyze Chen Yi's Happy Rain through the notion of poetic shi, therefore, is to consider the essential organizing force of Chinese aesthetic principles in contemporary compositions. As an analytical approach, this opens up possibilities both for listening and for naming. Examining a concept such as shi allows us to reconsider aspects of musical expression and experience that previously might have been lacking words for explication, or a framework for probing. ${ }^{(15)}$ To embrace Chinese aesthetic concepts as part of our analytical approach is a much-needed step. The integration of these concepts into contemporary musical practices did not just begin in the 1980s, when the newwave composers from Beijing and Shanghai arrived on the international scene. Rather, it has had a long history in America: its imprint is traceable to Chou Wen-chung's orchestral work The Fallen Petals (1953), composed more than sixty years ago, as well as to his notion of remerger (W. Chou 1978).

Nancy Yunhwa Rao

Music Department, Mason Gross School of the Arts

Stephanie Morris Marryott Music Building

Rutgers, the State University of New Jersey

81 George Street

New Brunswick, NJ 08901-1411

nancy.rao@rutgers.edu

\section{Works Cited}

Almén, Byron. 2006. "Modes of Analysis." Theory and Practice 31: 1-38.

Boyce, Conal. 1980. "Recitation of Chinese Poetry." Journal of the American Oriental Society 100 (4): 503-9.

Bush, Susan, and Hsio-yen Shih, eds. 2012. Early Chinese Texts on Painting. Hong Kong University Press. 
Chao, Yuan Ren. 1956. “Tone, Intonation, Singsong, Chanting, Recitative, Tonal Composition, and Atonal Composition in Chinese." In For Roman Jakobson: Essays on the Occasion of His Sixtieth Birthday, comp. Morris Halle, 52-59. Mouton \& C.

Chen, Yi. 2014. Happy Rain on a Spring Night. Theodore Presser Co.

2019. “Truly a Wenren-Remembering Chou Wen-chung (1923-2019)." NewMusicBox, November 3, 2019. https://nmbx.newmusicusa.org/truly-a-wenren-remembering-chou-wen-chung1923-2019/.

Chen, Yi, Marianne Kielian-Gilbert, Nancy Rao, John Roeder, and Jennifer Bain. 2020. “In Dialogue with Chen Yi: Compositional Images, Techniques, and Influences." Transcribed by Zackary Harrison. Edited transcript of the question and answer period that concluded a session on Chen Yi's music, November 3, 2017, sponsored by the Committee on the Status of Women at the 2017 Society for Music Theory annual meeting in Arlington, VA. http://hdl.handle.net/10222/79583.

Chou, Shan. 1991. “Tu Fu's Social Conscience: Compassion and Topicality in His Poetry.” Harvard Journal of Asiatic Studies 51 (1): 5-53.

Chou, Wen-Chung, 1978. "Towards a Re-merger in Music." In Contemporary Composers on Contemporary Music, eds. Elliott Schwartz and Barney Childs, 309-15. Da Capo Press.

Cox, Arnie. 2001. "The Mimetic Hypothesis and Embodied Musical Meaning." Musicae Scientiae 5 (2): 195-209.

2011. “Embodying Music: Principles of the Mimetic Hypothesis." Music Theory Online 17 (2). https://mtosmt.org/issues/mto.11.17.2/mto.11.17.2.cox.html

Egan, Ronald C. 1994. Word, Image, and Deed in the Life of Su Shi. Harvard University Press.

2004. "Nature and Higher Ideals in Texts on Calligraphy, Music, and Painting." In Chinese Aesthetics: The Ordering of Literature, the Arts, and the Universe in the Six Dynasties, ed. Zongqi Cai, 277-309. University of Hawai'i Press.

Everett, Yayoi Uno. 2007. "Calligraphy and Musical Gestures in the Late Works of Chou Wen-chung." Contemporary Music Review 26 (5-6): 578-81.

Fisk, Craig. 1986. “Literary Criticism.” In The Indiana Companion to Traditional Chinese Literature, ed. William H. Nienhauser, Jr., 49-58. Indiana University Press.

Fong, Wen C. 1999. "Chinese Calligraphy: Theory and History." In The Embodied Image: Chinese Calligraphy from the John B. Elliott Collection, eds. Robert E. Harrist, Jr. and Wen C. Fong, 28-84. Princeton University Press.

Hatten, Robert. 2004. Interpreting Musical Gestures, Topics, and Tropes: Mozart, Beethoven, Schubert. Indiana University Press.

2012. "Musical Forces and Agential Energies: An Expansion of Steve Larson's Model." Music Theory Online 18 (3). https://mtosmt.org/issues/mto.12.18.3/mto.12.18.3.hatten.html

Jullien, François. 1999. The Propensity for Things: Toward a History of Efficacy in China. Translated by Janet Lloyd. MIT Press.

Kao, Yu-Kung. 1991. “Chinese Lyric Aesthetics." In Words and Images: Chinese Poetry, Calligraphy, and Painting, eds. Alfreda Murck and Wen C. Fong, 47-90. Princeton University Press.

Kao,Yu-Kung and Tsu-lin Mei. 1971. "Syntax, Diction, and Imagery in T'ang Poetry." Harvard Journal of Asiatic Studies 31: 49-136.

Lai, Eric. 2018. "Musical Brushstrokes: Calligraphy and Texture in Chou Wen-chung's Music." In Polycultural Synthesis in the Music of Chou Wen-chung, eds. Mary I. Arlin and Mark A. Radice, 86- 
121. Routledge.

Lam, Lap. 2010. "Cultural Identity and Vocal Expression: The Southern School Tradition of. Poetry Chanting in Contemporary Guangzhou." Chinese Literature: Essays, Articles, Reviews 32: 23-52.

Larson, Steve. 2012. Musical Forces: Motion, Metaphor, and Meaning in Music. Indiana University Press.

Rao, Nancy Yunhwa. 2000. “The Role of Language in Music Integration in Chen Qigang's Poème Lyrique II." Journal of Music in China 2 (2): 270-91.

2016. “The Transformative Power of Rhythm and Gesture: Transnational Inflection in Chen Yi's Symphony No. 2." In Analytical Essays on Music by Women Composers: Concert Music, 19602000, eds. Laurel Parsons and Brenda Ravenscroft, 127-52. Oxford University Press.

2020. “Kalligraphie und Tinte in der zeitgenössischen chinesischen Musik: Der Fall Wen Deqing." Musik \& Ästhetik 93: 5-26.

Robinson, Douglas. 2017. Critical Translation Studies. Routledge.

Watson, Burton, trans. 2003. Selected Poems of Du Fu. Columbia University Press.

Wu, Chi-Jen. 2017. “Su Dongpo's Nian Nu Jiao in Cursive Calligraphy.” YouTube Video, 8:21. Jun 24, 2017. https://www.youtube.com/watch?v=ofJisKYwaco.

Xiao, Chi 蕭馳. 2003. Zhongguo sixiang yu shuqing chuantong: Wang Fuzhi shixue fawei 中國思想與抒情傳 統: 王夫之詩學發微 (Chinese Thought and Lyrical Tradition: Elucidating Wang Fuzhi's Theory of Poetry). Shanghai Guji Publisher.

Yung, Bell. 1989. Cantonese Opera: Performance as Creative Process. Cambridge University Press.

\section{Footnotes}

1. In addition to Everett 2007, discussion in this regard can also be found in Lai 2018.

Return to text

2. Chou Wen-chung summarized his view about the relationship between calligraphy and music in "Excerpts from a letter to Yayoi Uno Everett regarding Chinese calligraphy, 2006" http://www.chouwenchung.org/philosophy/calligraphy.php. Many composers have explored the relationship between Chinese calligraphic brushstroke and contemporary music. For a discussion of different approaches, see Rao 2020.

Return to text

3. For more detailed discussion of syntax in Chinese classic poetry, as well as the connection to poetic meaning, see Kao and Mei 1971.

Return to text

4. Chi Xiao, a Singapore scholar, has written extensively about the relationship between Du Fu's poetry and the notion of shi (see Xiao 2003). His use of shi as a concept to consider Chinese lyrical tradition has been widely cited. With regard to the notion of human consciousness in Chinese poetry, scholar Craig Fisk noted, rather than mimesis, "the object of representation is mood at a point in time and correspondences between mind and state of the surrounding world" $(1986,49)$. Return to text

5. In her discussion, Chen Yi labels three primary motives, A1, A2, A3. Musical Image 1 in my analysis corresponds with her motive A3. As will be seen later, Ascending Motion I in my analysis corresponds with her A2.

Return to text

6. I would strongly recommend that the pianist bring out the distinctive timbre/rhythm of the third musical image to give it a fresh sound, rather than underscoring its similarity with the first musical 
image and therefore sounding repetitive.

Return to text

7. One of the most famous long horizontal paintings is Along the River During the Qingming Festival by the Song dynasty artist Zhang Zeduan (1085-1145). It extends from crop fields and unhurried rural folk in bucolic scenery to the urban area in a scroll of 17.22 feet.

Return to text

8. The significant role of $C \#$ in the overall pitch structure piques my curiosity. During the Q\&A section of the panel, Chen Yi confirmed offers a rationale based on instrumentation. "I also used this $C$ sharp because the violin could play it loudly and brightly as a harmonic. When you play the third harmonic on an open string it's sharp; it's light and brighter." Chen further discussed the close relationship between image and instrumentation in her work. See Chen et al. 2020.

Return to text

9. In traditional Chinese education, children learn to recite a considerable number of classic poems by ear, and poetry recitation is fundamental to cultural education. Overall, the convention of classic poem recitation includes a wide range of practices and terminologies. Words such as yin 吟 (hum, intone), song 誦 (recite), yinsong 吟誦 (recite/chant), and yinchang 吟唱 (chant/sing) all fall under the notion of recitation of poetry. While they elude clear definitions, there are implied differences in degree of melodiousness and theatricality, evenness of duration, registral range, modes of delivery and so forth. English terms "recitation" and "chanting" are used here to connote the practice interchangeably. See Boyce 1980; Lam 2010; Chao 1956.

Return to text

10. Elsewhere I have discussed in greater detail the concept, patterns and usage of "heightened speech" from Beijing opera in contemporary Chinese composition. See Rao 2000. Bell Yung (1989) explores in great detail the tonal inflection of Chinese language and its relation to music.

Return to text

11. Although "source" normally refers to a text or specific objects, here it is used to refer to the practice of recitation.

Return to text

12. As an accomplished violinist growing up and enduring through the Cultural Revolution, Chen $\mathrm{Yi}$ is well versed in the sonority of the erhu, also called the "Chinese violin," and undoubtedly draws inspiration from the general style and performing tradition, perhaps even subconsciously. Return to text

13. It also connects to the notion of shi, as the thin and fragile timbre of the violin portrays a kind of musical effect akin to the aesthetic effect of dry ink strokes in the cursive calligraphy style.

Return to text

14. Many contemporary Chinese composers active on the international stage today were under the tutelage of composers such as Olivier Messiaen (Qigang Chen), György Ligeti (Xiaoyong Chen), and Mario Davidovsky (Chen Yi, Zhou Long, Lei Liang).

Return to text

15. Though a comparative study is not the purpose of this article, the concept of shi has some overlap with metaphors of musical force that have a long history within Western European music. The metaphors concern momentum created primarily (though not exclusively) by tonal harmony and melody. Recent examples include Larson 2012 and Hatten 2012. For further discussion on the concept of shi, its connection to Chinese calligraphy, and its relevance to the works of other contemporary Chinese composers, see Chen et al. 2020.

Return to text

\section{Copyright Statement}

Copyright (C) 2020 by the Society for Music Theory. All rights reserved. 
[1] Copyrights for individual items published in Music Theory Online (MTO) are held by their authors. Items appearing in MTO may be saved and stored in electronic or paper form, and may be shared among individuals for purposes of scholarly research or discussion, but may not be republished in any form, electronic or print, without prior, written permission from the author(s), and advance notification of the editors of MTO.

[2] Any redistributed form of items published in MTO must include the following information in a form appropriate to the medium in which the items are to appear:

This item appeared in Music Theory Online in [VOLUME \#, ISSUE \#] on [DAY/MONTH/YEAR]. It was authored by [FULL NAME, EMAIL ADDRESS], with whose written permission it is reprinted here.

[3] Libraries may archive issues of MTO in electronic or paper form for public access so long as each issue is stored in its entirety, and no access fee is charged. Exceptions to these requirements must be approved in writing by the editors of MTO, who will act in accordance with the decisions of the Society for Music Theory.

This document and all portions thereof are protected by U.S. and international copyright laws. Material contained herein may be copied and/or distributed for research purposes only.

Prepared by Lauren Irschick, Editorial Assistant

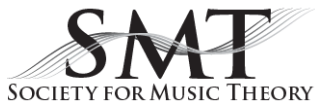

